\title{
Smoothness Measure for Image Fusion in Discrete Cosine Transform
}

\author{
Radhika Vadhi \\ Research Scholar, University \\ College of Engineering \\ JNTUK, Kakinada A.P., India
}

\author{
Veera Swamy Kilari \\ Professor, QIS College of \\ Engineering \& Technology, \\ Ongole, A.P., India
}

\author{
Srinivas Kumar Samayamantula \\ Professor, University College of \\ Engineering \\ JNTUK, Kakinada A.P., India
}

\begin{abstract}
The aim of image fusion is to generate high-quality images using information from source images. The fused image contains more information than any of the source images. Image fusion using transforms is more effective than spatial methods. Statistical measures such as mean, contrast, and variance, are used in Discrete Cosine Transform (DCT) for image fusion. In this paper, we use statistical measures, such as the smoothness of a block in the transform domain, to select appropriate blocks from multiple images to obtain a fused image. Smoothness captures important blocks in images and duly eliminates noisy blocks. Furthermore, we compare and analyze all statistical measures in the DCT domain. Experimental results establish the superiority of our proposed method over state-of-the-art techniques for image fusion.
\end{abstract}

Keywords-smoothness; statistical measures; DCT; image fusion

\section{INTRODUCTION}

Today video surveillance is common in many public places such as hospitals, banks, offices, airports, military installations and other traffic control applications. The cameras are located in any corner of the place to capture the visuals. Proper visual information is capture in the center of the apex and at the edges and corners, where the camera located, have the poor quality of visuals due to the limited focal depth of optical lenses. Even though two or more cameras are located in various corners to capture depth, then also the problem of poor quality appears. To overcome this poor quality vision problem, Digital image fusion has been emerged.

Digital image fusion is a novel digital image processing technique that involves combining information from source images to form a single, final image. The fused image contains more relevant and accurate information than any of the source images, which are captured by visual sensors. The statistical measures involved are simple to compute, and play a vital role in identifying important information in images. The combination of transforms and statistical measures helps to identify vital information in the source images. Digital image fusion techniques can be classified into two domains: spatial domain and transform domain. Some researchers have proposed image fusion techniques that are implemented in the spatial domain $[2,3,4,5,6,9,10,11,12,24,25,26,36$, and 37]. Also the Fusion techniques based on multi scale decomposition are popular $[7,8,13,14,15,16,18,19,20,21$, 22, and 23]. These involve grouping source images by observing a parameter called the activity level measure. The summation can be finalized by selecting coefficients with higher activity levels. The fused image is finally obtained by performing inverse multiscale operations. Discrete Wavelet Transform (DWT) is a multi-resolution transform used to fuse images. In the DWT domain, the maximum absolute value of the corresponding decomposed band coefficient at each position is selected as the activity level [20]. In image fusion using shearlet transforms [28], regional variance, and regional average gradients, regional spatial frequency is considered for high-frequency sub-band coefficients, and regional characteristics are used for low-frequency coefficients.

Most spatial domain image fusion techniques are complex and time consuming, and are hence not appropriate for realtime applications. In most communication, data is compressed prior to transmission, and images are coded in the JPEG format at present. Discrete Cosine Transform (DCT) is used in JPEG. Hence, we use the DCT domain in this study.

DCT-based methods, such as DCT + average [8], DCT + contrast [8], DCT + Variance1, DCT + Variance + Consistency Verification [1], are popular in the area. Prevalent DCT-based methods suffer from undesirable side-effects, such as blurring and blocking artifacts. In this work, we propose DCT + Smoothness to identify relatively less noisy blocks in source images for image fusion.

The rest of this paper is organized as follows: In Section 2, we describe smoothness calculation in the DCT domain, and the proposed fusion algorithm is detailed in Section 3. We discuss the results of experiments to test the performance of our proposed method in Section 4, and offer our conclusions in Section 5.

\section{SMOOTHNESS CALCULATION IN DCT DOMAIN}

Noise is ever presents in digital images during image acquisition, coding, transmission, and processing. Smoothness measures the relative smoothness of intensity in a region. It is high for a region of constant intensity, and low for regions with large excursions in the values of its intensity levels. Smoothness is a statistical method used to select relatively less noisy blocks in image fusion. Hence, smoothness algorithms tend to be superior in performance than others. Smoothness attempts to capture important patterns in an image while leaving out noisy blocks. Since our technique is implemented in the DCT domain, it saves time and computational complexity if the fused image needs to be stored or 
transmitted in the JPEG format. Smoothness is high when variations in AC coefficient values are low, and vice versa.

A two-dimensional (2D) DCT transform of an $\mathrm{N} \times \mathrm{N}$ block of an image $f(m, n)$ is defined as

where $\mathrm{k}, \mathrm{l}=0,1, \ldots ., \mathrm{N}-1$, and

$$
\alpha(k)= \begin{cases}\frac{1}{\sqrt{2}} & \text { if } k=0 \\ 1, & \text { otherwise }\end{cases}
$$

In order to compute smoothness, the DC coefficient in Equation (1) needs to be eliminated to obtain results containing only AC coefficients because the DC coefficient is not useful in judging the smoothness of block

$$
F(k, l)=\frac{2 \alpha(k) \alpha(l)}{N} \times \sum_{m=0}^{N-1} \sum_{n=0}^{N-1} f(m, n) \times \cos \left[\frac{(2 m+1) \pi k}{2 N}\right] \times \cos \left[\frac{(2 n+1) \pi l}{2 N}\right]
$$

AC coefficients indicate variations in image blocks. The absolute value is considered to assign weight to all variations in $\mathrm{AC}$ coefficients. Hence, variations in the $z$ block are computed as follows:

$$
U(z)=\sum_{k, l}|F(k, l)| \quad \text { where } k \neq 0, l \neq 0
$$

In sum, the smoothness of the $z$ block can be exactly calculated from its DCT coefficients by the absolute sum of the AC coefficients of the DCT block. Here, a high value of $U(z)$ indicates that smoothness is low, and vice versa.

\section{PROPOSED FUSION ALGORITHM}

The details of image fusion are shown in Fig. 1, which depicts the common framework of a JPEG encoder combined with our proposed image fusion method. This method can be extended to any number of source images.

Our proposed fusion algorithm is as follows:

1) Consider two or more multi-focused source images.

2) Each source image is divided into sub-blocks

3) Apply 2D-DCT to each sub-block of each source image.

4) Variations in the DCT-transformed sub-bocks are calculated using Eq. (4).

5) The smoothness of each block is compared with that of a corresponding block from other source images.

6) The blocks with higher smoothness values are selected.
7) All sub-blocks are arranged into a single block.

8) $2 D$ - inverse DCT is applied to each sub-block of the fused image.

The general fusion procedure is explained below. The source images are divided into sub-blocks, and 2D-DCT is applied to each sub-block. The statistical measure (smoothness) is calculated for all 2D-DCT sub-blocks, which are subsequently chosen based on smoothness values for fusion. Inverse DCT is applied to each sub-block to convert them into pixels.

\section{EXPERIMENTAL RESULTS AND DISCUSSION}

In this section, we describe the results of experiments on the proposed statistical measure for image fusion, and compare them with results from other important image fusion methods in the literature. The images used in our experiment are shown in Fig. 3

\section{A. Measurement Criteria}

Several objective evaluation methods are available to assess image fusion performance. Mutual Information (MI) $[29,32]$ is an important one used to test the quality of the fused image, and involves calculating information common to source images $f_{1}, f_{2}$ and fused image $f_{S}$. Edge Strength and Orientation Preservation (ESOP) $\left(Q^{f_{1} f_{2} / f_{S}}\right)$ values can be calculated using Xydeas work $[30,33]$. Lin et al. proposed a Feature Similarity Index method (FSIM), [31] and Normalized Cross-Correlation (NCC) [27] is another measurement parameter for image fusion. The NCC is computed between the mean of the ground truth image/ benchmark and that of the fused image.

\section{B. Experimental Analysis}

The algorithms were executed on six standard images shown in Fig. 3. In general, there is a problem in considering the images to be fused. We created out-of-focus or multi-focus images by blurring parts of the original image using low-pass filters. Blurring can be carried out by convolution with a Gaussian to reduce detail. The amount of blurring was considered in comparison with spatial frequency and visibility as in [26]. The original image and blurred images are shown in Fig. 4.

We used sub-blocks of size $8 \times 8$. Mutual Information (MI) is a measure used to test image quality using quantity of information. ESOP $\left(\mathrm{Q}^{\mathrm{fl} / \mathrm{f} / \mathrm{fs}}\right)$ evaluates edge information, and FSIM is a metric for phase congruence and edge information between the source images and the fused image. The experimental results are listed in Table 1. 


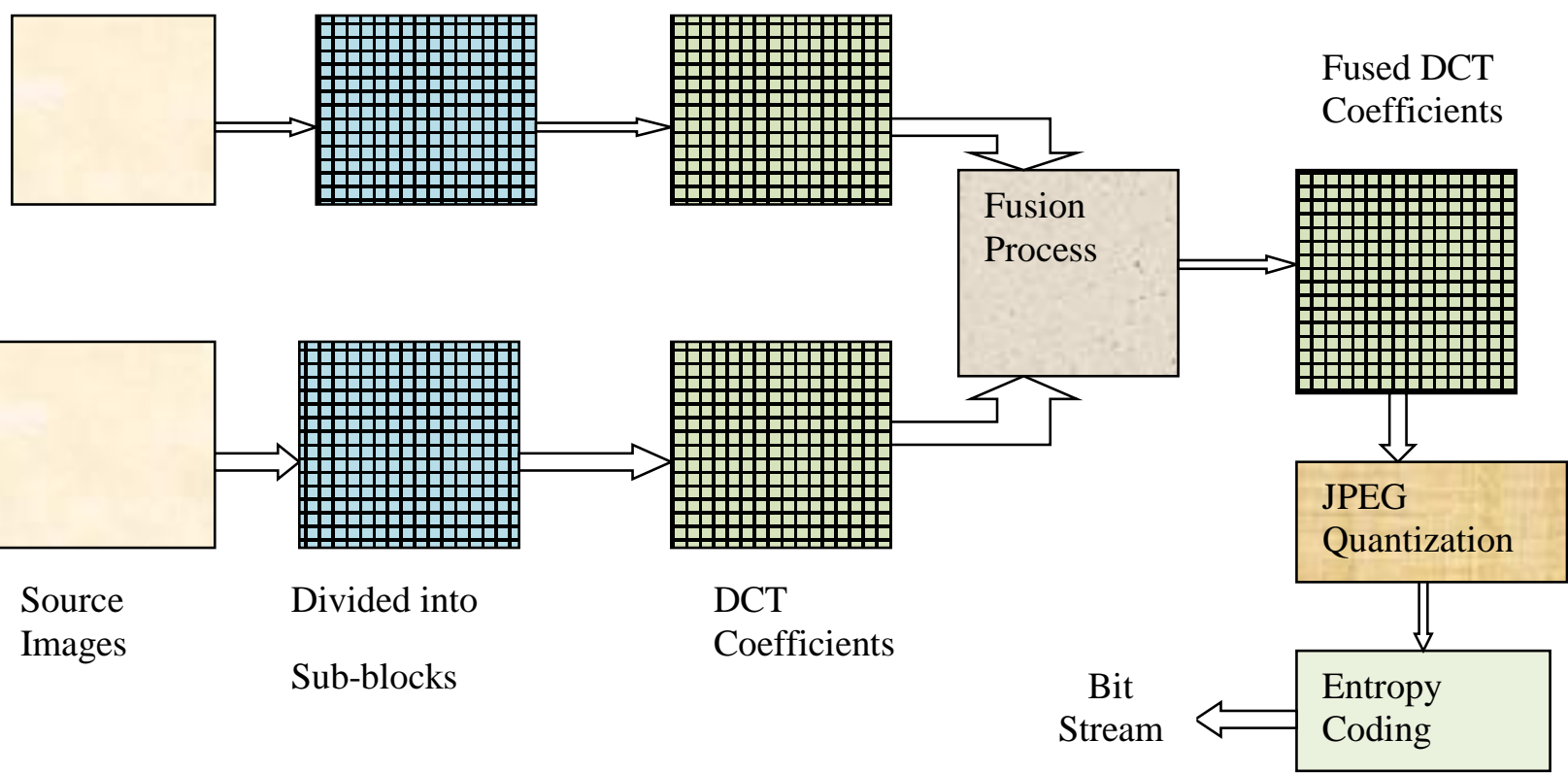

Fig. 1. General schematic representation of image fusion method

Fusion Process

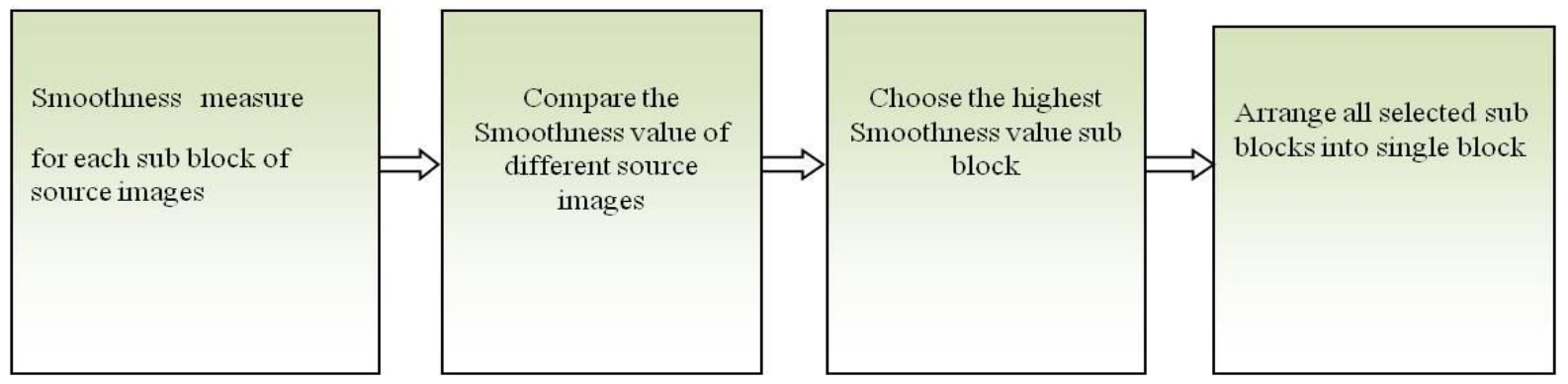

Fig. 2. Fusion process
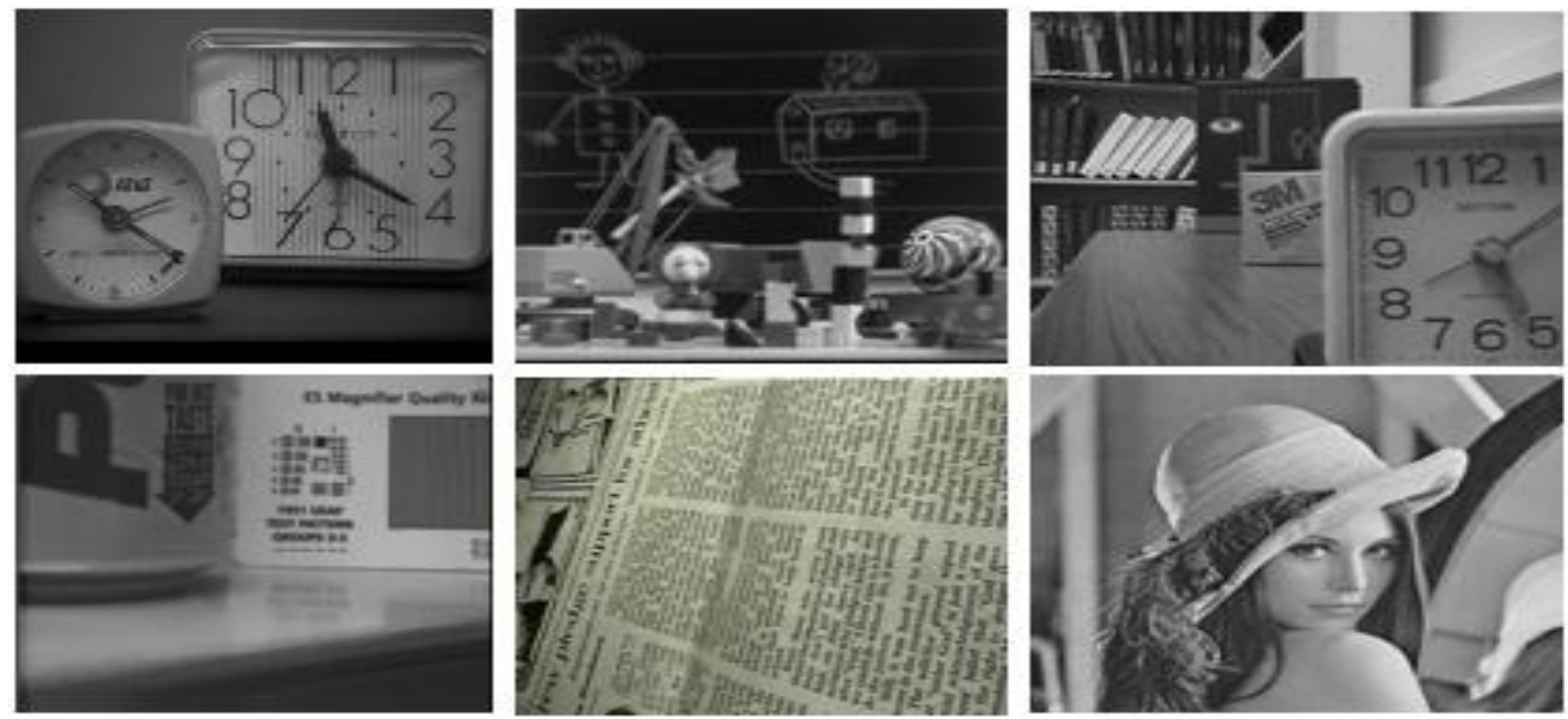

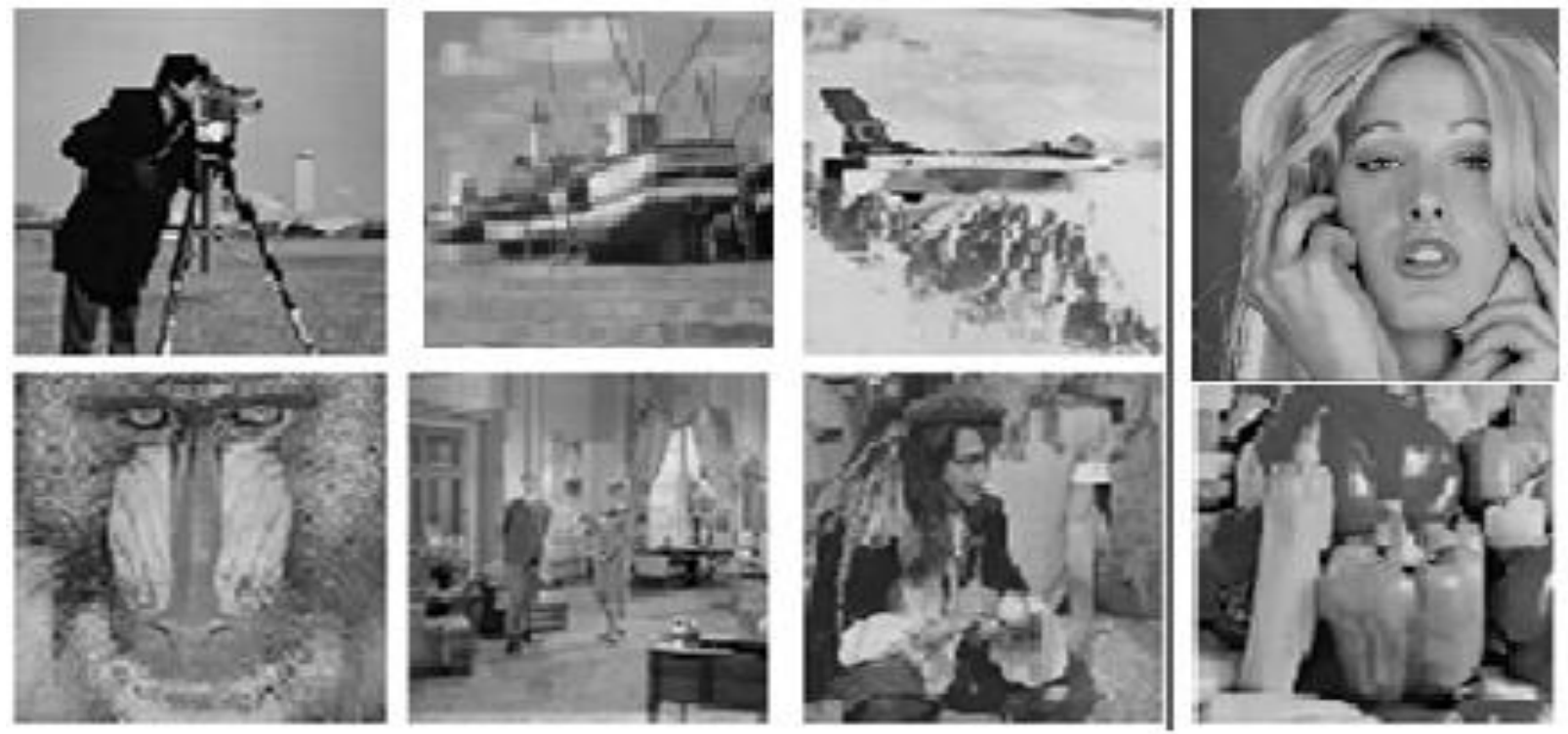

Fig. 3. Images used in experiment

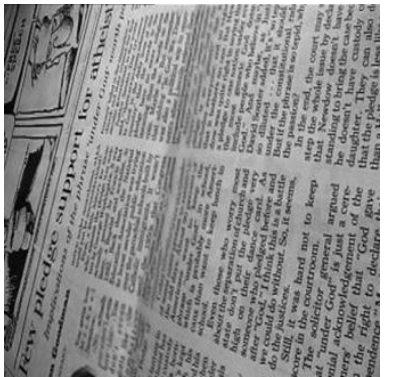

(a)

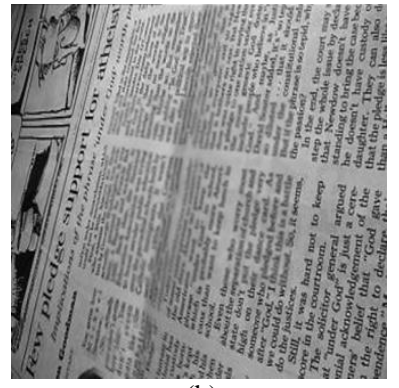

(b)

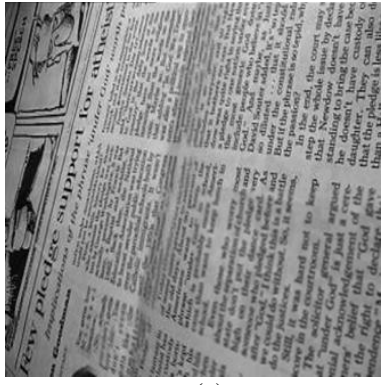

(c)

Fig. 4. Paper, (a) Original image (b) Left-side blurred image (c) right-side blurred image

TABLE I. OBJECTIVE ASSESSMENT OF IMAGE Fusion ALGORITHMS

\begin{tabular}{|l|l|l|c|c|c|}
\hline & Fusion Rule & MI & $\begin{array}{c}Q^{f_{1} f_{2} / f_{S}} \\
(\text { ESOP })\end{array}$ & FSIM \\
\hline Clock & DCT + Avg. & 4.3933 & 0.9050 & 0.9997 & 0.9991 \\
\hline & DCT + Contrast & 4.4910 & 0.9146 & 0.9997 & 0.9992 \\
\hline & DCT + Variance & 4.5347 & 0.9149 & 0.9997 & 0.9992 \\
\hline & DCT + Smoothness & $\mathbf{4 . 5 9 4 0}$ & $\mathbf{0 . 9 1 5 2}$ & $\mathbf{0 . 9 9 9 7}$ & $\mathbf{0 . 9 9 9 3}$ \\
\hline & DCT + Avg. & 3.2999 & 0.0402 & 0.9998 & 0.9979 \\
\hline & DCT + Contrast & 3.2803 & 0.8691 & 0.9996 & 0.9960 \\
\hline & DCT + Variance & 3.5650 & 0.8748 & 0.9998 & 0.9987 \\
\hline Disk & DCT + Smoothness & $\mathbf{3 . 8 1 4 3}$ & $\mathbf{0 . 8 7 8 5}$ & $\mathbf{0 . 9 9 9 9}$ & $\mathbf{0 . 9 9 9 1}$ \\
\hline & DCT + Avg. & 3.3177 & 0.0354 & 0.9996 & 0.9974 \\
\hline & DCT + Contrast & 3.9216 & 0.8958 & 0.9995 & 0.9978 \\
\hline & DCT + Variance & 4.1189 & 0.9012 & 0.9997 & 0.9983 \\
\hline & DCT+ Smoothness & $\mathbf{4 . 1 8 4 6}$ & $\mathbf{0 . 9 0 3 9}$ & $\mathbf{0 . 9 9 9 8}$ & $\mathbf{0 . 9 9 8 7}$ \\
\hline
\end{tabular}




\begin{tabular}{|c|c|c|c|c|c|}
\hline & DCT + Contrast & 3.9474 & 0.8995 & 0.9998 & 0.9986 \\
\hline & DCT + Variance & 4.4756 & 0.9138 & 0.9999 & 0.9994 \\
\hline & DCT+ Smoothness & 4.5236 & 0.9148 & 0.9999 & 0.9995 \\
\hline \multirow[t]{4}{*}{ Paper } & DCT + Avg. & 2.9544 & 0.0197 & 0.9994 & 0.9843 \\
\hline & DCT + Contrast & 3.1565 & 0.8497 & 0.9989 & 0.9688 \\
\hline & DCT + Variance & 3.9129 & 0.8947 & 0.9997 & 0.9828 \\
\hline & DCT+ Smoothness & 3.9306 & 0.8950 & 0.9997 & 0.9928 \\
\hline \multirow[t]{4}{*}{ Lena } & DCT + Avg. & 3.7464 & 0.0477 & 0.9997 & 0.9973 \\
\hline & DCT + Contrast & 3.9497 & 0.8925 & 0.9997 & 0.9979 \\
\hline & DCT + Variance & 4.2831 & 0.8919 & 0.9998 & 0.9984 \\
\hline & DCT+ Smoothness & 4.3085 & 0.8928 & 0.9987 & 0.9999 \\
\hline \multirow[t]{4}{*}{ Cameraman } & DCT + Avg. & 3.6970 & 0.0264 & 0.9996 & 0.9973 \\
\hline & DCT + Contrast & 3.7701 & 0.8440 & 0.9993 & 0.9973 \\
\hline & DCT + Variance & 4.1361 & 0.8733 & 0.9997 & 0.9984 \\
\hline & DCT+ Smoothness & 4.1795 & 0.8748 & 0.9998 & 0.9986 \\
\hline \multirow[t]{4}{*}{ Woman } & DCT + Avg. & 3.6814 & 0.0336 & 0.9998 & 0.9963 \\
\hline & DCT + Contrast & 3.7791 & 0.8720 & 0.9997 & 0.9941 \\
\hline & DCT + Variance & 4.3559 & 0.8893 & 0.9999 & 0.9986 \\
\hline & DCT +Smoothness & 4.4202 & 0.8919 & 0.9999 & 0.9989 \\
\hline \multirow[t]{4}{*}{ F17 } & DCT + Avg. & 3.5011 & 0.0205 & 0.9997 & 0.9978 \\
\hline & DCT + Contrast & 3.5892 & 0.8983 & 0.9996 & 0.9974 \\
\hline & DCT + Variance & 3.8001 & 0.8987 & 0.9998 & 0.9981 \\
\hline & DCT+ Smoothness & 3.8786 & 0.9017 & 0.9998 & 0.9986 \\
\hline \multirow[t]{4}{*}{ Fishingboat } & DCT + Avg. & 3.2273 & 0.0244 & 0.9998 & 0.9985 \\
\hline & DCT + Contrast & 3.2305 & 0.8857 & 0.9996 & 0.9982 \\
\hline & DCT + Variance & 3.4229 & 0.8889 & 0.9998 & 0.9990 \\
\hline & DCT+ Smoothness & 3.5059 & 0.8934 & 0.9999 & 0.9994 \\
\hline \multirow[t]{4}{*}{ Mandrill } & DCT + Avg. & 3.4635 & 0.0554 & 0.9995 & 0.9909 \\
\hline & DCT + Contrast & 3.5544 & 0.8765 & 0.9993 & 0.9806 \\
\hline & DCT + Variance & 4.4831 & 0.9206 & 0.9998 & 0.9963 \\
\hline & DCT+ Smoothness & 4.4875 & 0.9205 & 0.9998 & 0.9962 \\
\hline \multirow[t]{4}{*}{ Livingroom } & DCT + Avg. & 3.2742 & 0.0341 & 0.9996 & 0.9933 \\
\hline & DCT + Contrast & 3.4754 & 0.8553 & 0.9994 & 0.9894 \\
\hline & DCT + Variance & 4.2227 & 0.8830 & 0.9997 & 0.9966 \\
\hline & DCT+ Smoothness & 4.2728 & 0.8844 & 0.9998 & 0.9969 \\
\hline \multirow[t]{4}{*}{ Pirate } & DCT + Avg. & 3.5956 & 0.0335 & 0.9997 & 0.9963 \\
\hline & DCT + Contrast & 3.9354 & 0.8598 & 0.9996 & 0.9949 \\
\hline & DCT + Variance & 4.5513 & 0.8891 & 0.9998 & 0.9982 \\
\hline & DCT+ Smoothness & 4.6094 & 0.8910 & 0.9999 & 0.9985 \\
\hline \multirow[t]{3}{*}{ Peppers } & DCT + Avg. & 4.3024 & 0.0238 & 0.9999 & 0.9994 \\
\hline & DCT + Contrast & 3.2305 & 0.8996 & 0.8857 & 0.9982 \\
\hline & DCT + Variance & 4.5821 & 0.9193 & 0.9998 & 0.9994 \\
\hline
\end{tabular}




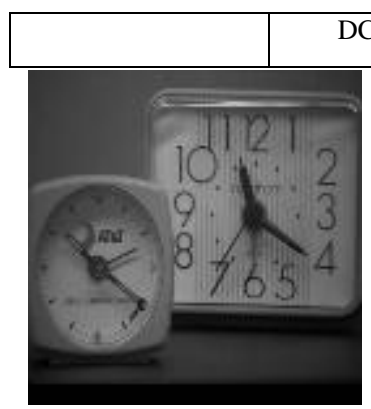

(a)

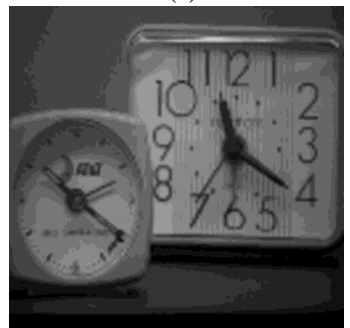

(e)

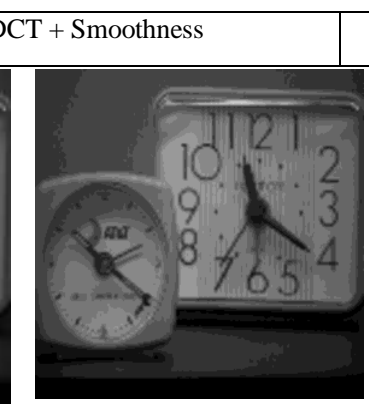

(b)

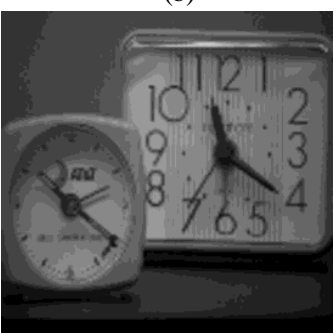

(f)

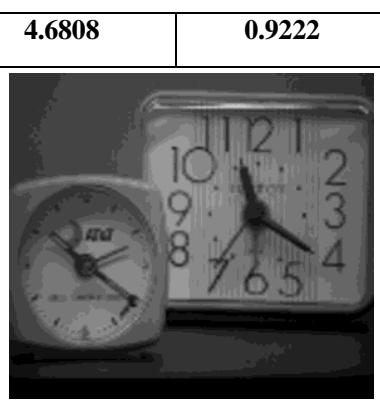

(c)

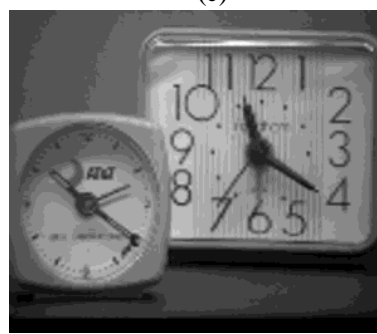

(g)

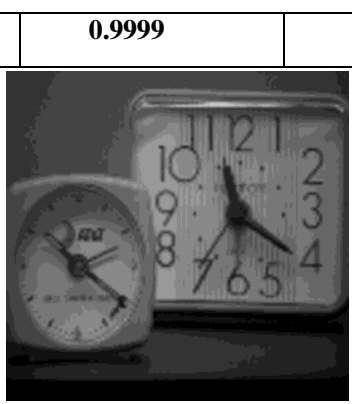

(d)

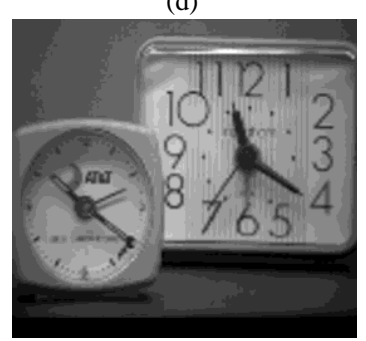

(h)

Fig. 5. "Clock." (a) Ground truth. (b) Left-blurred image. (c) Right-blurred image. (d) DCT + Avg. (e) DCT + Contrast. (f) DCT + Variance. (g) DCT + Smoothness. (h) Shearlet transform

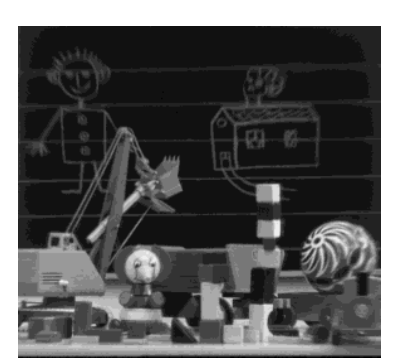

(a)

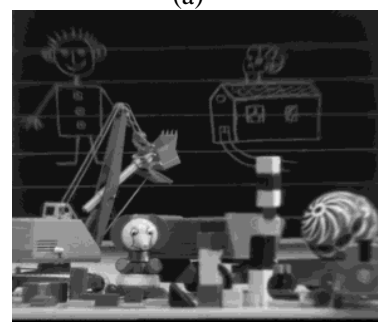

(e)

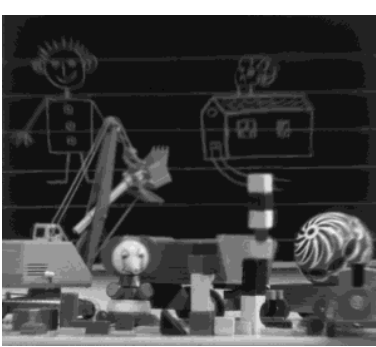

(b)

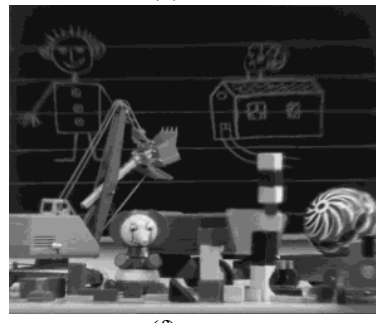

(f)

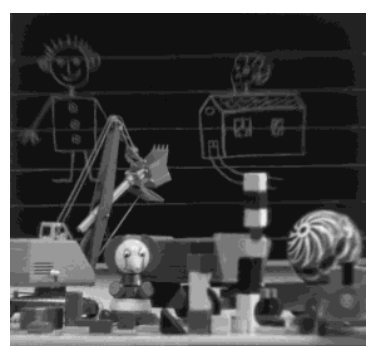

(c)

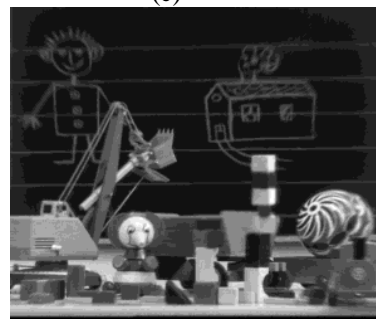

(g)

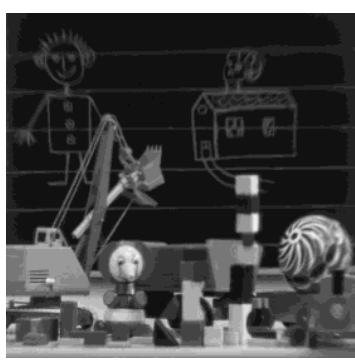

(d)

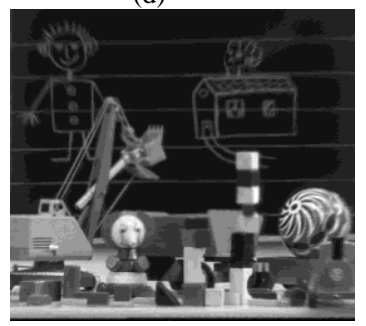

(h)

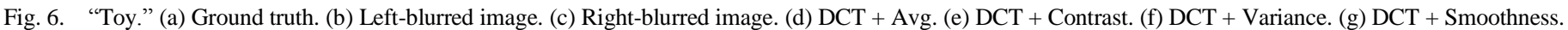
(h) shearlet transform

TABLE II. RUNTIME VALUES OF VARIOUS ALGORITHMS FOR “CLOCK.”

\begin{tabular}{|l|c|c|c|}
\hline DCT + Avg. & DCT + Con. & DCT + Var. & DCT + Smoothness \\
\hline 4.741602 & 6.236915 & 4.650012 & 4.530772 \\
\hline
\end{tabular}

TABLE III. EXPERIMENTAL RESULTS (DCT AND SHEARLET TRANSFORM)

\begin{tabular}{|c|c|c|c|c|c|}
\hline & Fusion Rule & MI & $Q^{f_{1} f_{2} / f_{S}}$ & FSIM & $\mathrm{NCC}$ \\
\hline Clock & DCT+ Smoothness & $\mathbf{4 . 5 9 4 0}$ & 0.9152 & 0.9997 & 0.9993 \\
\hline & shearlet transform & 4.49190 & 0.9010 & 0.9943 & 0.9992 \\
\hline Toy & DCT+ Smoothness & 3.8143 & 0.8785 & 0.9999 & 0.9991 \\
\hline
\end{tabular}




\begin{tabular}{|c|c|c|c|c|c|}
\hline & shearlet transform & 3.0648 & 0.8602 & 0.9862 & 0.9973 \\
\hline Disk & DCT+ Smoothness & $\mathbf{4 . 1 8 4 6}$ & $\mathbf{0 . 9 0 3 9}$ & $\mathbf{0 . 9 9 9 8}$ & $\mathbf{0 . 9 9 8 7}$ \\
\hline & shearlet transform & 3.2895 & 0.8877 & 0.9875 & 0.9973 \\
\hline Pepsi & DCT+ Smoothness & $\mathbf{4 . 5 2 3 6}$ & $\mathbf{0 . 9 1 4 8}$ & $\mathbf{0 . 9 9 9 9}$ & $\mathbf{0 . 9 9 9 5}$ \\
\hline & shearlet transform & 3.9992 & 0.8901 & 0.9919 & $\mathbf{0 . 9 9 9 7}$ \\
\hline Paper & DCT+ Smoothness & $\mathbf{3 . 9 3 0 6}$ & $\mathbf{0 . 8 9 5 0}$ & 0.9828 & $\mathbf{0 . 9 9 2 8}$ \\
\hline & shearlet transform & 2.5694 & 0.8539 & $\mathbf{0 . 9 9 8 7}$ & $\mathbf{0 . 9 9 9 9}$ \\
\hline Lena & DCT+ Smoothness & $\mathbf{4 . 3 0 8 5}$ & $\mathbf{0 . 8 9 2 8}$ & 0.9797 \\
\hline
\end{tabular}

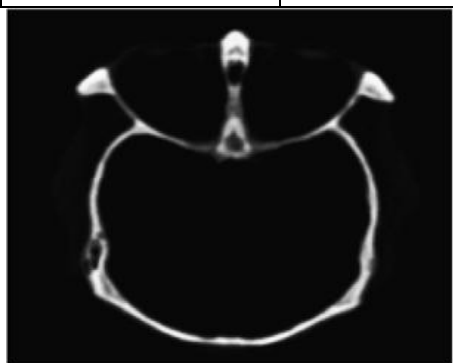

(a)

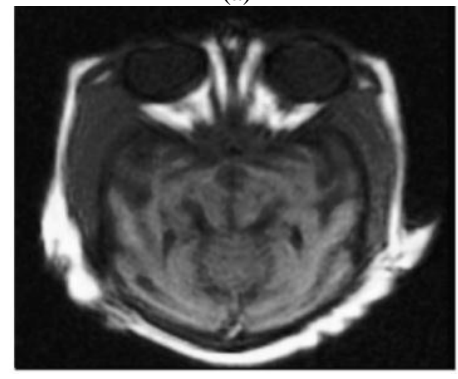

(d)

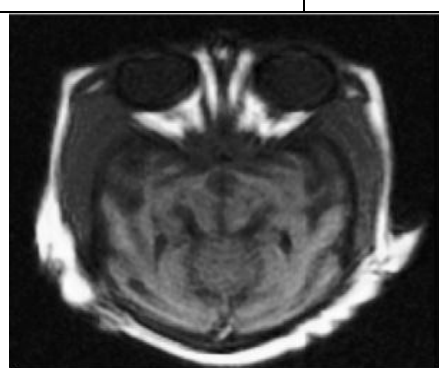

(b)

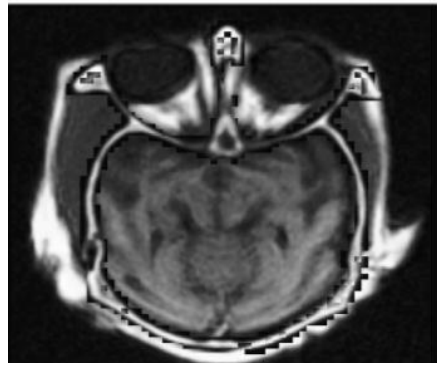

(e)

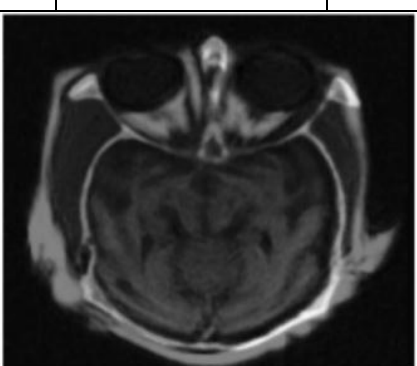

(c)

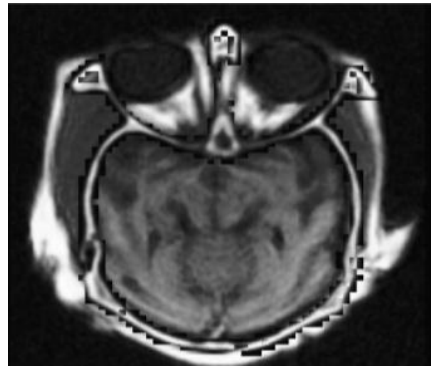

(f)

Fig. 7. "Medical images" (a) CT (b) MRI (c) DCT + Avg (d) DCT + Contrast (e) DCT + Variance (f) DCT + Smoothness

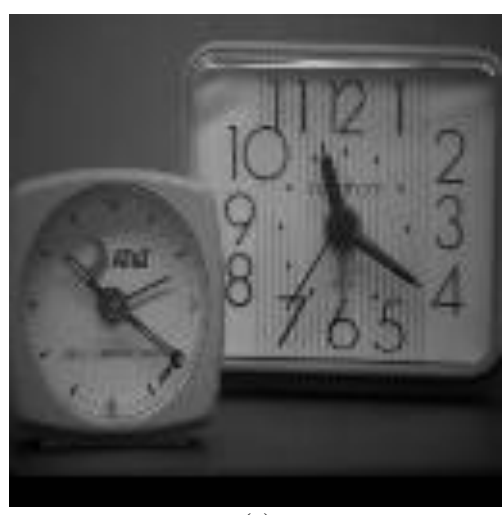

(a)

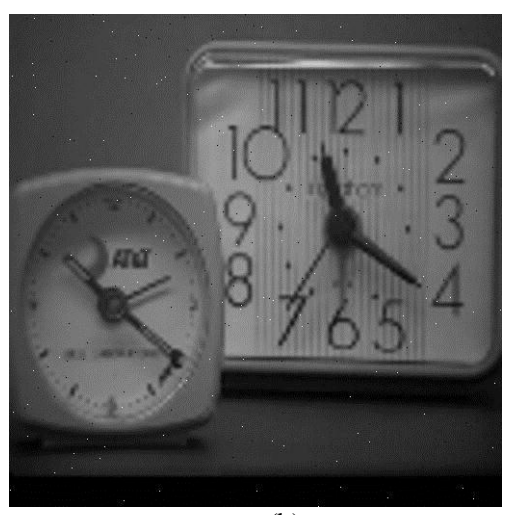

(b)

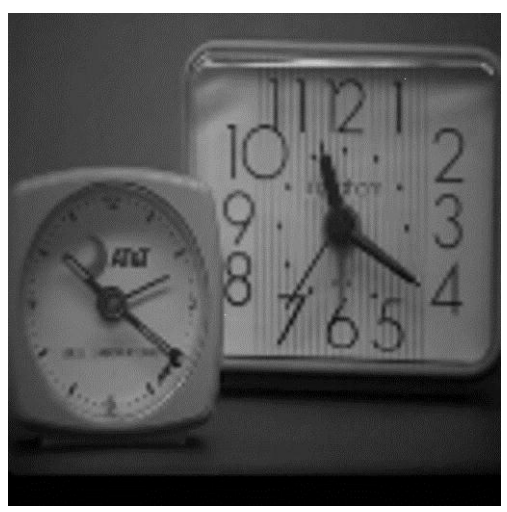

(c)

Fig. 8. Clock, (a) Original image (b) Noise image1 (c) Noise image 2

TABLE IV. EXPERIMENTAL RESUlTS (FOR NOISE IMAGES)

\begin{tabular}{|l|l|l|l|l|}
\hline & MI & ESOP & FSIM & NCC \\
\hline DCT+ Avg & 3.9190 & 0.9555 & 0.9992 & 0.9939 \\
\hline DCT+ Con & 3.9188 & 0.9528 & 0.9992 & 0.9942 \\
\hline DCT+ Var & 3.9180 & 0.9536 & 0.9990 & 0.9935 \\
\hline DCT+ Smoothness & $\mathbf{3 . 9 1 9 4}$ & $\mathbf{0 . 9 5 7 3}$ & $\mathbf{0 . 9 9 9 3}$ & $\mathbf{0 . 9 9 4 4}$ \\
\hline
\end{tabular}


The results in Table 1 show that MI, FSIM, and NCC improved with our proposed DCT + Smoothness approach. We can see that DCT + Smoothness is competent than the other DCT-based methods. The amount of blurriness was created by a [4 4] Gaussian filter, and its standard deviation is 6. The experimental results for the "clock" and "toy" images are shown in Fig. 5 and Fig. 6, respectively.

DCT + Avg., DCT + Contrast, and DCT + Variance are the existed algorithms. By carefully observing the fusion results, it is concluded that the method DCT + Average results blurring the fused image (Fig.5.d). The method DCT + Contrast and DCT + Variance results some blocking artifacts (Fig.5.e and Fig.5.f). There are ringing artifacts for the shearlet transform based fusion method (Fig.5.h). The time taken by each method to perform the fusion operation is shown in Table 2. The runtime of the proposed DCT + Smoothness was shorter than that of existing methods for image fusion. All algorithms were executed on a Pentium IV processor with $3 \mathrm{GHz}$ ROM, and $504 \mathrm{MB}$ of Random-Access Memory (RAM). The operating system used was Windows XP Professional 2002.

We also compared the image fusion performance of our method with that of the shearlet transform proposed by Liu and Wang[28]. Low-frequency sub-band coefficients were processed based on the energy of each sub-band, and highfrequency sub-band coefficients were processed based on the variance of each sub-band.

The results indicate that the smoothness measure in the DCT domain is suitable for selecting blocks for image fusion. This yields better results than the variance characteristic in the shearlet domain. The comparisons between DCT + Smoothness and Shearlet transforms are given in Table3. The proposed algorithm is also tested on noise images and naturally acquired images. The experimental results of CT, MRI are given in Fig.7. Experiments are also performed on Noise images. We created noise images by considering impulse noise with different densities. The original image and noise images are given in Fig.8.

\section{CONCLUSIONS}

In this paper, we proposed a method for image fusion that used smoothness as a statistical measure in the DCT domain, and experimentally compared it with other methods that employ different statistical measures. The experiments established the superiority of our smoothness-based measure in the DCT domain in terms of complexity and execution time. Our method was also superior when compared with multiresolution transform-based image fusion methods. It is thus more appropriate for real-time applications.

\section{AKNOWLEDGEMENTS}

Thank you to the www.fusion org and http://utopia.duth.gr/ nmitiano/fusion.html; http://www.hindawi.com/journals/mpe/2009/128317/fig10/; http://www.ece.lehigh.edu/SPCRL/IF/disk.htm

http://utopia.duth.gr/ nmitiano/fusion.html]

https://www.pantechsolutions.net/basics-of-image-fusion] for providing the source images.

\section{REFERENCES}

[1] M. B. A. Haghighat, A. Aghagolzadeh, and H. Seyedarabi, "Multi-focus image fusion for visual sensor networks in DCT domain," Computers \& Electrical Engineering. 37(5), 789-797 (2011).

[2] A. A. Goshtasby and S. Nikolov, "Image fusion: Advances in the state of the art," Information Fusion. 8(2), 114-118 (2007).

[3] V. S. Petrovic and C. S. Xydeas, "Gradient-based multiresolution image fusion," IEEE Transactions on Image Processing. 13(2), 228-237 (2004).

[4] T. Stathaki, Image fusion algorithms and applications, Academic Press, (2008).

[5] N. Mitianoudis and T. Stathaki, "Pixel-based and region-based image fusion schemes using ICA bases," Information Fusion. 8(2), 131-142 (2007).

[6] H. Li, B. Manjunath and S. Mitra, "Multisensor image fusion using the wavelet transform," Graphical Models and Image Processing. 57(3), 235-245 (1995).

[7] J. Tang, "A contrast based image fusion technique in the DCT domain," Digital Signal Processing. 14(3), 218-226 (2004).

[8] G. Piella, "A general framework for multiresolution image fusion: from pixels to regions," Information Fusion. 4 (4), 259-280 (2003).

[9] Y. Xia and H. Leung, "A fast learning algorithm for blind data fusion using a novel L2-norm estimation," IEEE Sensors Journal. 14(3), 666672 (2014).

[10] M. B. A. Haghighat, Ali Aghagolzadeh, and Hadi Seyedarabi, "Realtime fusion of multi-focus images for visual sensor networks," 6th Iranian Conference on Machine Vision and Image Processing (MVIP). 1-6, (2010).

[11] H. Liu, J. Yang, Z. Wu, and Q. Zhang, "Fast single image dehazing based on image," Journal of Electronic Imaging. New. 24(1), 013020013020 (2015).

[12] X.q. Luo, Z.C. Zhang, and X.j. Wu, "Adaptive multistrategy image fusion method," Journal of Electronic Imaging.23 (5), 053011 (2014).

[13] O. Rockinger, "Image sequence fusions using a shift-invariant wavelet transform," Proceedings of IEEE International Conference on Image Processing. 3, 288-291 (1997)

[14] R. C. Gonzalez, R. E. Woods, and S. L. Eddins, Digital Image Processing Using MATLAB. Low price edition, 2002.

[15] A. Saleem, A. Beghdadi, and B. Boashash, "Image fusion-based contrast enhancement," EURASIP Journal on Image and Video Processing. 1-17 (2012).

[16] B. Yang and S. Li, "Pixel-level image fusion with simultaneous orthogonal matching pursuit," Information Fusion. 13(1), 10-19 (2012).

[17] H. Li, B. S. Manjunath, and S. K. Mitra. "Image Fusion Using the Wavelet Transform," Proc. First International Conference on Image Processing ICIP 94, Austin, Texas. 1, 51-55 (1994).

[18] D. Drajic and N. Cvcjic, "Adaptive fusion of multi-model surveillance image sequences in visual sensor networks," IEEE Trans Consum Electron. 8(2), 119-30 (2007).

[19] G. Bhatnagar and B. Raman, "A new image fusion technique based on directive contrast," Electron Letter on Computer Vision and Image Analysis. 8(2), 18-38 (2009).

[20] Y. B.-Shoshan and Y. Yitzhaky, "Improvements of image fusion methods," Journal of Electronic Imaging. 23(2), 023021 (2014).

[21] Q. Yuan, L. Zhang, and H. Shen, "Hyperspectral image denoising with a spatial-spectral view fusion strategy," IEEE Transaction on Geoscience and Remote Sensing. 52(5), $2314-2325$ (2014).

[22] T. Peli and E. Peli, "Contrast in complex images," J. Op. Soc. Am. A 7. 2030-2040 (1990).

[23] R. Maruthi and Sankarasubramanian, "Multi-focus image fusion based on the information level in the regions of the images" Journal of Theoretical and Applied information Technology. 3(4), 80-85 (2007).

[24] V. Radhika, V. Swamy Kilari, and S. Kumar Samayamantula, "Uniformbased approach for image fusion," ICECCS-2012, CCIS305, SpringerVerlag Berlin Heidelberg. 186-194 (2012). 
[25] X. Liu and J. Wang, "Image fusion based on shearlet transform and regional features," International Journal of Electronics and Communications (AEÜ). 68(6), 1-7 (2013).

[26] M. B. A. Haghighat, A. Aghagolzadeh, and H. Seyedarabi, "A nonreference image fusion metric based on mutual information of image features," Computers \& Electrical Engineering. 37(5), 744 -756 (2011).

[27] G. Piella and Heijmans, "New quality measures for image fusion," Polytechnical University of Catalonia (UPC). Jordi Girona, 08034 Barcelona, Spain. 1-3 (2003).
[28] L. Zhang and X. Mou, "FSIM: A feature similarity index for image quality assessment," IEEE transactions on Image Processing. 20(8), 2378-2386 (2011).

[29] G. H. Qu and D. L. Zhang, "Information measure for performance of image," Electronic Letters. 38(7), 313-315 (2002).

[30] C. S. Xydeas and V. Petrovic, "Objective image fusion performance measure," Electronic Letters. 36(4), 308-309 (2000). 